\title{
LA CONSERVACION, PROTECCION Y MEJORAMIENTO DEL MEDIO AMBIENTE, UNO DE LOS FINES DE LA EDUCACION COLOMBIANA EN LOS ALBORES DEL SIGLO XXI
}

PATRICIA CASTRILLON A. Socióloga

Centro de Estudios Ambientales C.E.A. Universidad Autónoma de Manizales

A n f o r a

\section{INTRODUCCION}

a educación en Colombia se rige por los principios contemplados en la Constitución Política Nacional, en la Ley General de la Educación, en la Ley que organiza el servicio público de la Educación Superior y en sus respectivos decretos reglamentarios.

La Constitución Colombiana define la 
educación como: un derecho de la persona y un servicio público con función social. La educación busca que todas las personas accedan al conocimiento, a la ciencia y a la cultura; formar en el respeto a los derechos humanos, a la paz y a la democracia, en el trabajo y la recreación, para el mejoramiento cultural y la protección del medio ambiente.

Al mismo tiempo la Constitución define como derechos colectivos y del ambiente el derecho a un ambiente sano y a la participación de la comunidad en las decisiones que puedan afectarlo. el derecho a la protección estatal de la diversidad e integridad ambientales y a la conservación de las áreas de especial importancia ecológica, el derecho a la planificación del manejo $y$ aprovechamiento de los recursos naturales que garanticen su desarrollo sostenido y su conservación y el derecho a la protección estatal del espacio público y su destinación al uso común.

Las Leyes hacen operativa la Constitución, en lo que respecta al medio ambiente la Ley 99 de 1993 y sus decretos reglamentarios, establecen los derechos y los deberes de los colombianos en
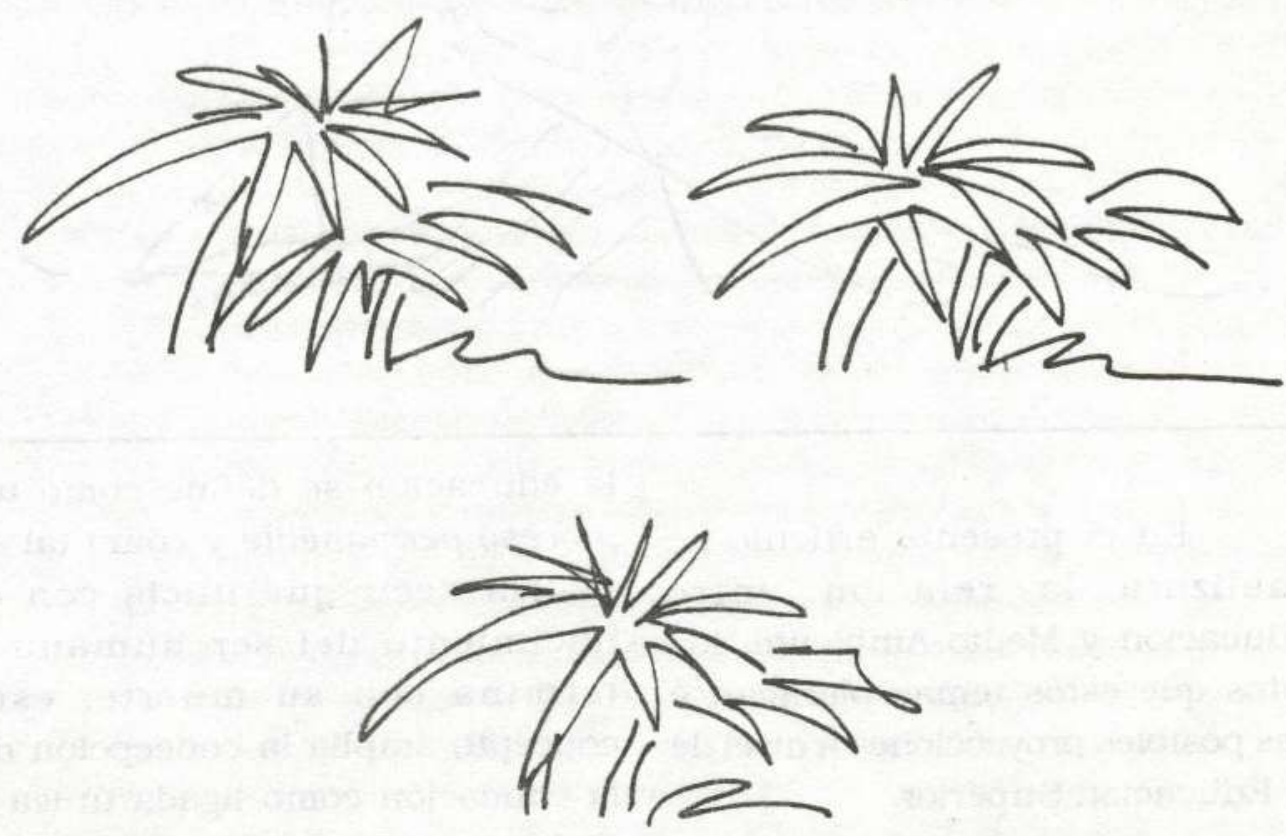
relación con el medio ambiente. Pero esta no es la única Ley que La educación es definida en define el marco de las relaciones entre los colombianos y el medio ambiente, existen otras leyes que regulan conductas comportamientos que tienen que ver con el tema, tales como las Leyes de la Educación, las Leyes Sanitarias, entre otras.

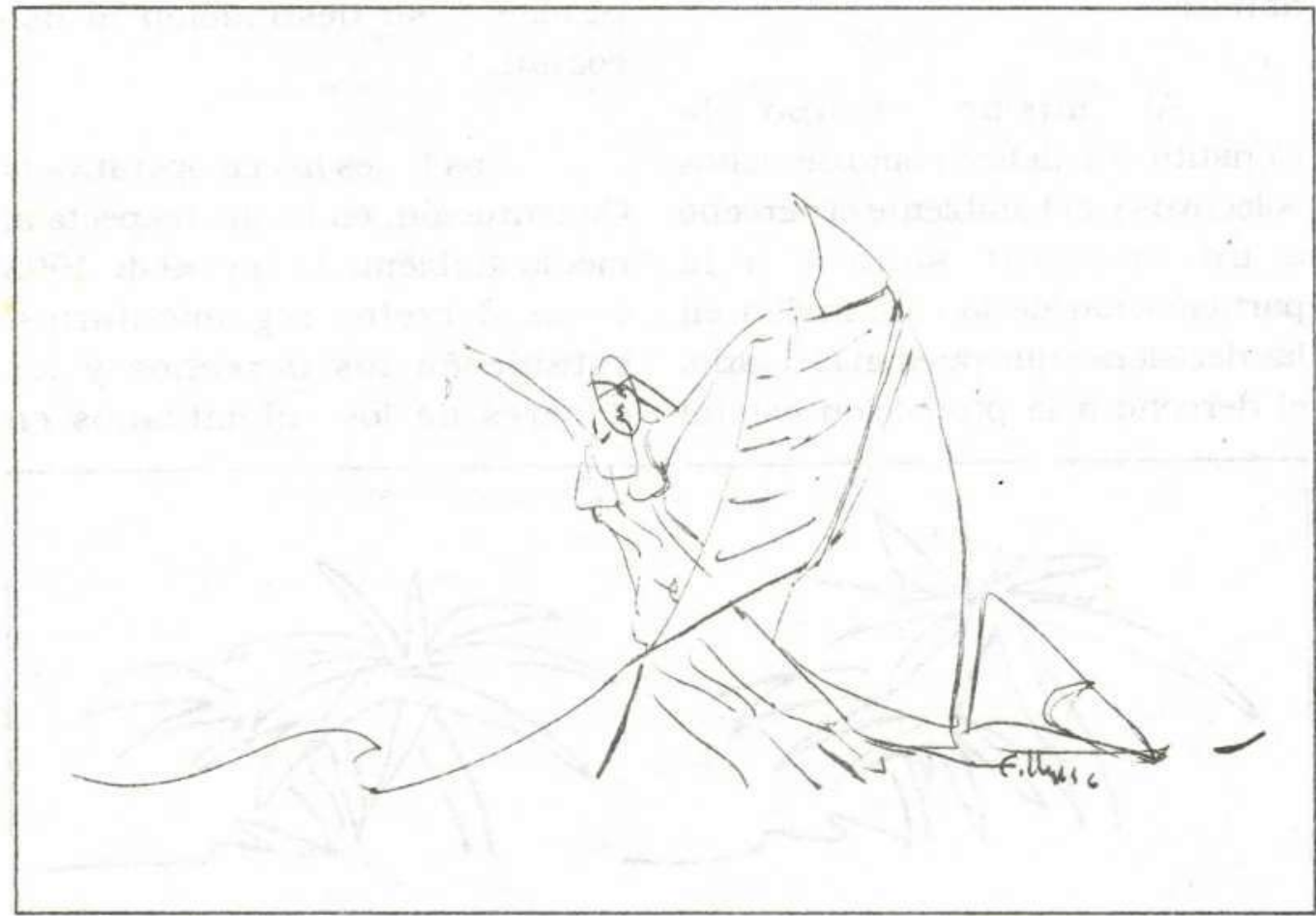

la educación se define como un

En el presente artículo se analizará la relación entre Educación y Medio Ambiente, los retos que estos temas plantean y sus posibles proyecciones a nivel de la Educación Superior.

\section{EL CONCEPTO DE EDUCACION} la Ley como «un proceso de formación permanente, personal, cultural y social que se fundamenta en una concepción integral de la persona humana, de su dignidad, de sus derechos y sus deberes».

Lo primero a destacar es que

proceso permanente y como tal se podría decir que inicia con el nacimiento del ser humano y termina con su muerte: este concepto amplía la concepción de la educación como ligada única y exclusivamente a una actividad que se desarrolla en los claustros y se 
abre a todos y cada uno de los espacios donde el ser humano crece y se desarrolla.

Definirlo como un proceso de formación implica más que una simple transmisión y asimilación de información, en tanto acción y efecto de formar o formarse, la educación se convierte en el acto educa a nadie») fuertemente condicionado por las motivaciones. intereses, necesidades y valores de las personas. Es un hecho demostrado que las personas memorizan más fácilmente aquellas informaciones que consideran necesarias o interesantes o simplemente útiles.

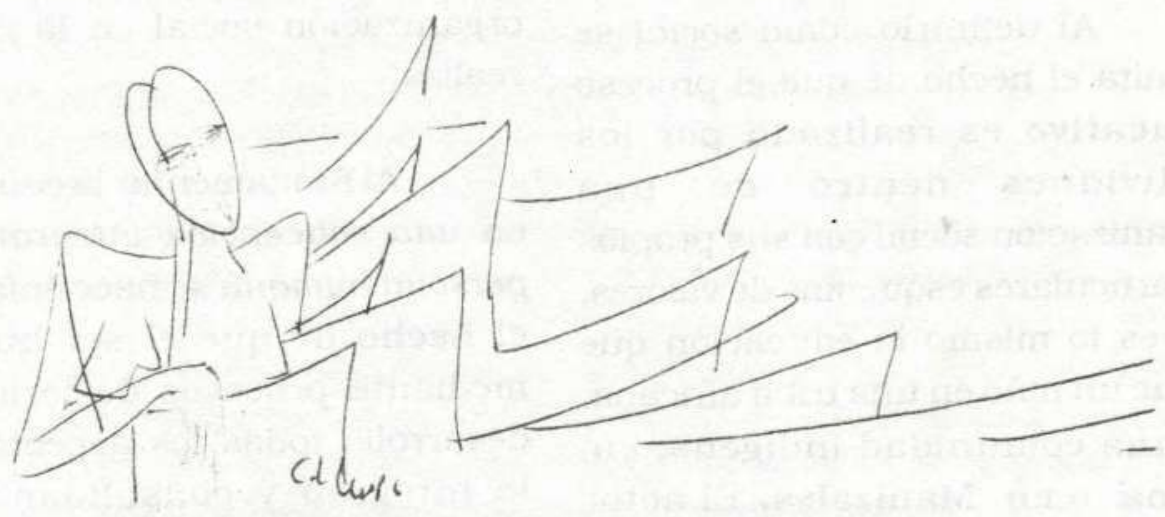

mediante el cual se da la forma al ser humano, es decir, se hace y se desarrolla como persona.

Finalmente al ser cultural y social se enfatiza el contenido y el

Al ser personal se enfatiza el hecho de que la educación, que ocurre a través de procesos de aprendizaje, es un acto individual (madie aprende por nadie» $y$ «nadie objeto de este proceso. En efecto. en el acto educativo se transmiten los conocimientos, los valores y las destrezas alcanzadas por un grupo. por una comunidad, en un país $e$ 
incluso por el ser humano como especie. En estos momentos y gracias a los avances logrados en las comunicaciones y en la informática, se dispone de un cúmulo considerable de información. Este simple hecho implica un cambio en las destrezas y aptitudes requeridas por las personas, más que memorizar se requiere desarrollar la capacidad de análisis crítico de la información, la capacidad de seleccionar aquella útil y aplicable a la realidad en la cual se interactúa.

Al definirlo como social se resalta el hecho de que el proceso educativo es realizado por los individuos dentro de una organización social con sus propios y particulares esquemas de valores. No es lo mismo la educación que sigue un niño en una tribu africana. en una comunidad indígena, en China o en Manizales. El acto

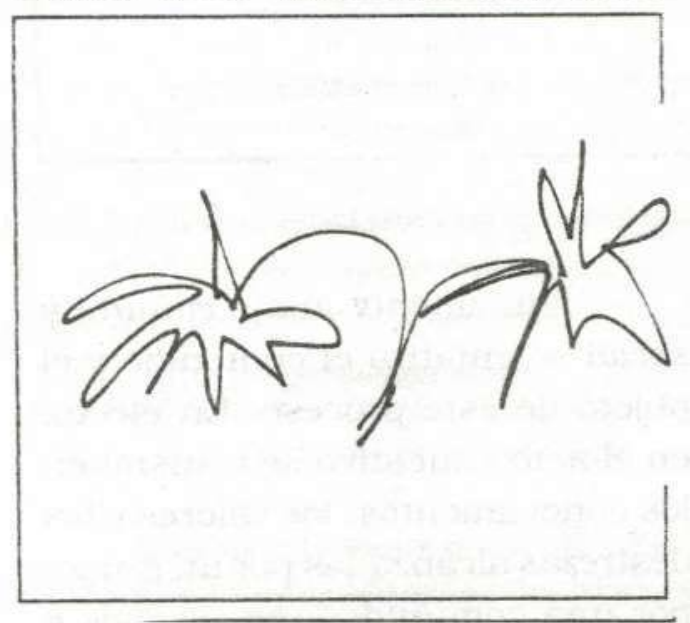

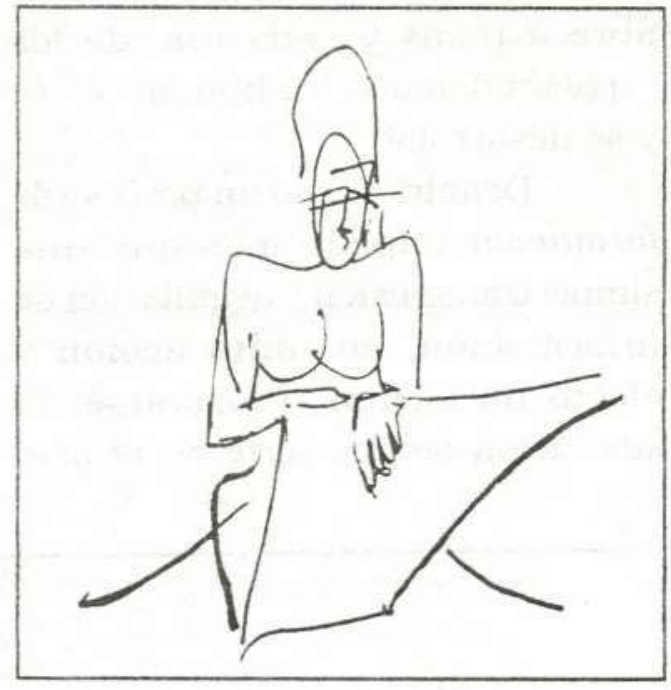

educativo está condicionado en su forma y en su fondo por la organización social en la cual se realiza.

Al fundamentar la educación en una concepción integral de la persona humana se hace énfasis en el hecho de que el ser humano mediante procesos de formación desarrolla todas los aspectos que lo integran y consolidan como persona.

Al fundamentarla en la dignidad de la persona humana, se reconoce el derecho inherente de todas las personas al respeto.

Finalmente, al fundamentar la educación en los derechos y deberes de la persona humana, se hace explícito el carácter social de dicho proceso. puesto que dichos 




deberes y derechos no son construcciones abstractas $y$ absolutas sino reglas de juego elaboradas dentro de un orden jurídico determinado.

\section{LA EDUCACIÓN Y EL AMBIENTE}

\subsection{La ley General de la Educación}

La Ley General de la Educación formula en forma explícita trece fines, el décimo de ellos tiene una relación directa con el medio ambiente

Se considera un fin de la educación en Colombia «la adquisición de una conciencia para la conservación, protección y mejoramiento del medio ambiente. de la calidad de la vida, del uso racional de los recursos naturales. de la prevención de desastres. dentro de una cultura ecológica y del riesgo, y la defensa del patrimonio cultural de la Nación».

El medio ambiente incluye no sólo el entorno natural sino el social en el cual se desarrollan los seres vivos. La cultura ecológica implica el cultivo de valores basados en el conocimiento científico de las relaciones entre los seres vivos y el medio ambiente en que viven $y$ se desarrollan y en el respeto a la vida en todas sus manifestaciones.

Desde este punto de vista, la Educación Ambiental se encuentra estrechamente relacionada con otros fines definidos a la educación tales como la formación en el respeto a la vida, el acceso al

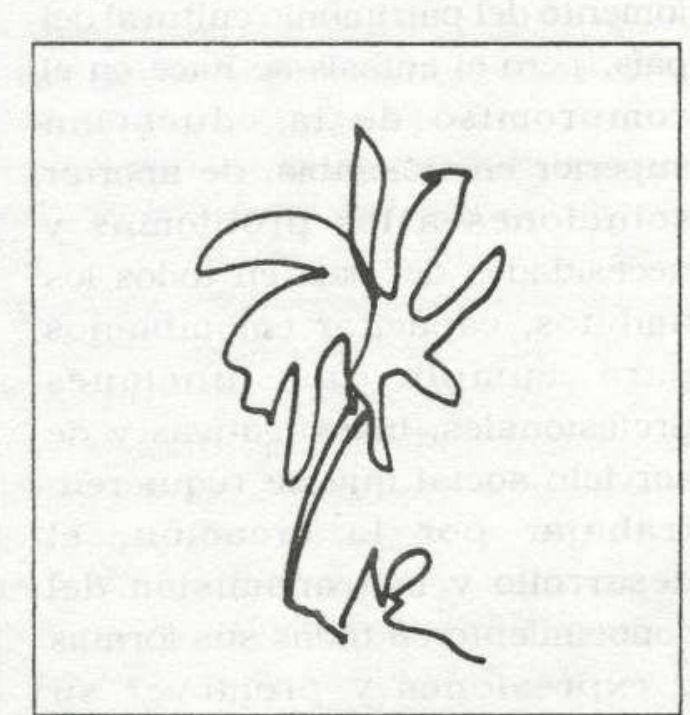


conocimiento, la ciencia, la técnica y demás bienes y valores de la cultura, el estudio y la comprensión crítica de la cultura nacional y de la diversidad étnica y cultural del país, con fundamento de la unidad nacional, entre otros.

\subsection{La Ley de la Educación} Superior (2).

Le Ley fija diez objetivos a la Educación Superior en Colombia. el noveno de ellos tiene que ver con el medio ambiente:

«Promover la preservación de un medio ambiente sano y fomentar la educación y cultura ecológica».

\section{Otros}

objetivos complementarios a la formación en materia medio ambiental, tienen que ver con la conservación y el fomento del patrimonio cultural del país, pero el énfasis se hace en el compromiso de la educación superior en Colombia, de aportar soluciones a los problemas y necesidades del país en todos los ámbitos, capacitar colombianos para cumplir las funciones profesionales, investigativas y de servicio social que se requieren. trabajar por la creación, el desarrollo y la transmisión del conocimiento en todas sus formas $\mathrm{y}$ expresiones y promover su

utilización en todos los campos para solucionar las necesidades del país, ser factor de desarrollo científico, cultural, económico. político y ético a nivel nacional y regional.

\section{LA EDUCACION AMBIENTAL EN LAS UNIVERSIDADES.}

3.1. Un programa de Pregrado, un Programa de Posgrado, o una asignatura obligatoria u optativa dentro de un Plan de Estudios.

Actualmente la Educación Ambiental en las Universidades podría clasificarse básicamente en programas de pregrado. en programas de postgrado o en asignaturas obligatorias 0 selectivas dentro de un plan de estudios determinado.

Esta educación se caracteriza por impartir una serie de informaciones y conocimientos relacionados con el medio ambiente, generalmente en cátedras de ecología, tratando el tema de forma asilada, como un compartimiento más de los que se abordan durante los años de formación.

Este estilo de educación si bien es necesaria dentro del proceso

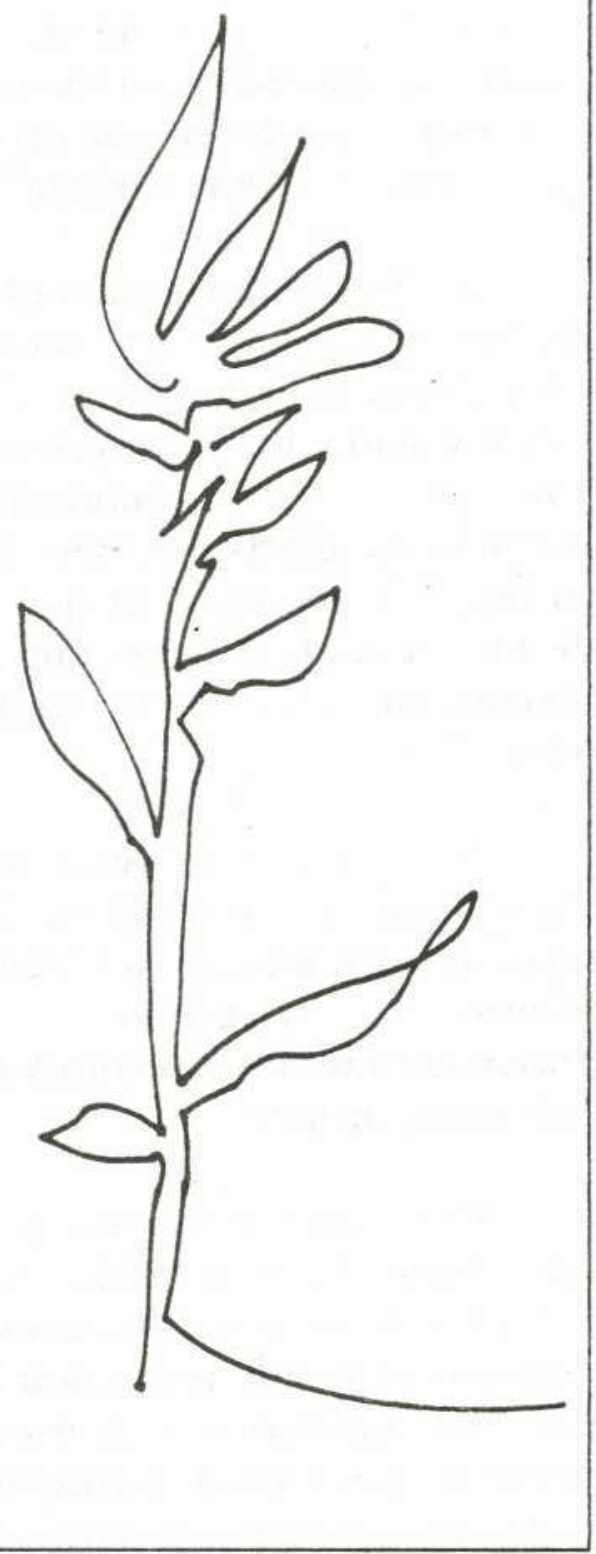


educativo, no es suficiente para lograr los fines y objetivos que le han sido trazados al proceso educativo en materia medio ambiental; de un lado porque como programa opcional, no es accesible a todos los estudiantes y de otro lado porque el tiempo dedicado al tema durante un semestre o durante un seminario, parece corto para abordar los temas que tienen que ver con el medio ambiente.

La Educación Ambiental, objetivo y fin de la educación colombiana, debe garantizarse a todos y cada uno de quienes conforman la comunidad universitaria, independientemente del programa de estudios que se adelante y de la función que se desempeñe dentro de dicha comunidad.

\subsection{La Educación Ambiental} como tema transversal en los Currículos Universitarios y como práctica integrada al funcionamiento de los centros de educación superior.

Uno de los caminos propuestos para alcanzar los objetivos trazados al proceso educativo en lo que respecta a la temática ambiental y a otras temáticas tales como la formación en valores cívicos y para la convivencia, entre otros, es su tratamiento como tema transversal dentro de los diferentes currículos de todos los programas educativos tanto formales, como no formales e informales que se impartan en un centro de formación superior.

Asi planteado el tema parece fácil y evidente su solución, si dictar una cátedra o contar con un programa de pregrado o de postgrado en Medio Ambiente no es suficiente para garantizar el cumplimiento de los fines y objetivos trazados a la educación superior en materia medio ambiental, entonces integremos el tema en todas las cátedras y




programas del centro educativo y asunto resuelto.

La pregunta siguiente es cómo hacerlo? y con quiénes?

Aún los países que han iniciado acciones en este campo no cuentan con respuestas y soluciones definitivas. Se puede afirmar que el tratamiento transversal de temas complejos en las diferentes asignaturas y cátedras está en experimentación.

Existen experiencias que sirven de marco de referencia pero aún no hay acuerdos sobre metodologías. contenidos.

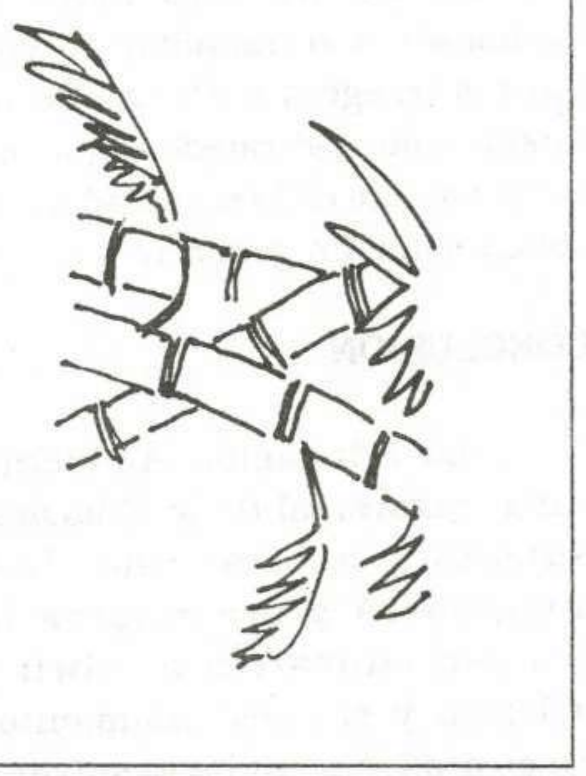

procesos. El camino está abierto a la innovación, particularmente porque todo proceso educativo en general y la educación en materia medio ambiental en particular, tienen un componente cultural y social especifico que se debe considerar al momento de querer aplicar fórmulas y procedimientos generales, o que han funcionado en otras culturas y sociedades.

Lo que sí parece claro hasta ahora es el hecho de que la Educación Ambiental debe atravesar todas y cada una de las cátedras y actividades que se realizan en un centro educativo, la Educacion Ambiental debe incorporarse en las diferentes asignaturas pero también en la forma en que se organiza y funciona. la institución como la casa, deben brindar en todos y cada uno de sus lugares físicos, espacios para el aprendizaje de nuevos valores y actitudes hacia el medio ambiente.

Las instituciones deben brindarle a su comunidad estudiantil la posibilidad de desarrollar actitudes y aptitudes que favorezcan una relación de respeto consigo mismo. con los demás y con el medio ambiente. incluyendo en este proceso no sólo a los alumnos o educando sino 

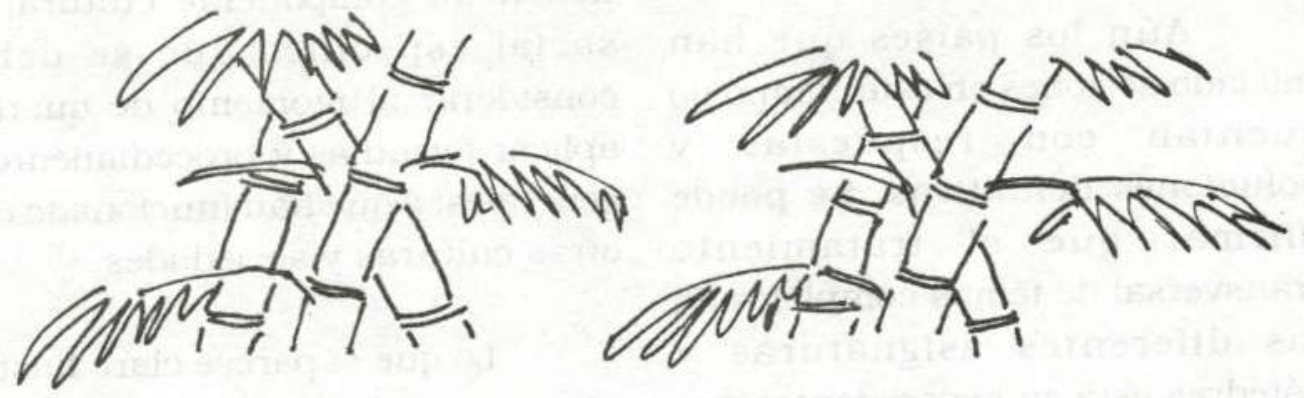

también a los educadores y a toda la comunidad universitaria en general incluyendo a directivos y padres de familia.

En efecto, la Educación Ambiental es un proceso de formación integral, que exige nuevos modelos de relación e interacción con nosotros mismos. con los demás y con la naturaleza. Cómo podemos pretender educar en la no violencia y en el respeto cuando no se garantizan relaciones de respeto en la familia, en el aula, en la calle?

La Educación Ambiental como proceso integrador y facilitador de las relaciones de los seres vivos entre ellos y la naturaleza exige coherencia, visión integradora y práctica. Aspectos que han faltado.en la formación tradicional y que pueden explicar el hecho de que las personas pasen por un centro educativo sin formarse como ciudadanos y como sujetos íntegros y éticos. De otro modo, cómo se puede explicar el estado actual de la sociedad no sólo colombiana sin general?

\section{CONCLUSION}

La Educación Ambiental, tema transversal de la Educación Superior, aporta una línea integradora y orientadora del proceso educativo al abrir la reflexión y el cuestionamiento a preguntas que debe resolver la 
comunidad educativa en su conjunto.

En dónde estamos? Planeta Tierra. País, región. municipio, ciudad barrio.

Cuáles son los principales problemas que tenemos? Diagnósticos Sectoriales y Territoriales.

- Cuáles son las posibles soluciones que vemos? Políticas vigentes y Planes Nacionales. Departamentales y Municipales de Desarrollo.

Cómo puede contribuir la Universidad a la solución de la problemática identificada?
Posicionamiento institucional.

Qué responsabilidad le compete a los futuros profesionales?

Qué responsabilidad le compete al personal directivo?

Qué responsabilidad tienen los padres de familia?

Qué responsabilidad le compete a la Sociedad Civil y al Estado?

Estas son sólo algunas de las preguntas que pueden formularse antes de iniciar un proceso de Educación Ambiental que básicamente debe contribuir a facilitar el conocimiento integral de la realidad en la que vivimos, para poder mejorarla. $\bigcirc$ 